\title{
Microbiota development in early life mediates association between mode of delivery and vaccine responses
}

Authors: E.M. de Koff $\mathrm{MD}^{1,2}$, D. van Baarle $\mathrm{PhD}^{3,4}$, M.A. van Houten $\mathrm{PhD}^{1,5}$, M. Reyman $\mathrm{PhD}^{2,6}$, G.A.M. Berbers $\mathrm{PhD}^{3}$, F. de Heij MSc${ }^{3}$, M.L.J.N. Chu BSc${ }^{2}$, E.A.M. Sanders $\mathrm{PhD}^{2,3}$, D. Bogaert $\mathrm{PhD}^{2,3,7 \# *}, \mathrm{~S}$. Fuentes $\mathrm{PhD}^{3 \#}$

\section{Affiliations:}

1. Spaarne Academy, Spaarne Gasthuis, Hoofddorp and Haarlem, Netherlands

2. Department of Paediatric Immunology and Infectious Diseases, Wilhelmina Children's Hospital and University Medical Centre Utrecht, Utrecht, Netherlands

3. Centre for Infectious Disease Control, National Institute for Public Health and the Environment, Bilthoven, Netherlands

4. Department of Medical Microbiology and Infection prevention, Virology and Immunology research Group, University Medical Centre Groningen, Groningen, Netherlands

5. Department of Paediatrics, Spaarne Gasthuis, Hoofddorp and Haarlem, Netherlands

6. Department of Dermatology, Erasmus University Medical Centre, Rotterdam, Netherlands

7. Medical Research Council and University of Edinburgh Centre for Inflammation Research, Queen's Medical Research Institute, University of Edinburgh, Edinburgh, UK

\# Co-senior authors

* Corresponding author: Prof. dr. Debby Bogaert, 47 Little France Crescent, Edinburgh, EH16 4TJ, United Kingdom, tel. +44 (0) 131242 6352, d.bogaert@ed.ac.uk.

Word count main text: 3924 words 


\begin{abstract}
The gut microbiota in early life, when critical immune maturation takes place, may influence the immunogenicity of childhood vaccinations. We assessed the association between mode of delivery, gut microbiota development in the first year of life, and mucosal antigen-specific immunoglobulin $\mathrm{G}(\mathrm{IgG})$ responses against pneumococcal and meningococcal conjugate vaccination at ages 12 and 18 months, respectively, in a prospective birth cohort of 120 infants. Birth by natural delivery was associated with higher IgG responses against both vaccines, which for the anti-pneumococcal IgG response could be explained by a gut microbial community composition with high abundances of Bifidobacterium and Escherichia coli in the first weeks of life. High E. coli abundance in the same period was also associated with higher anti-meningococcal IgG responses. Our results suggest that associations between mode of delivery and antibody responses to routine childhood vaccines are mediated by gut microbiota development.
\end{abstract}

Word count: $144 / 150$ words 


\section{Introduction}

Vaccination in early childhood is estimated to save millions of lives each year ${ }^{1}$. Vaccine-induced protection is mediated through a combination of innate, humoral and cellular immunity, and is often quantified by measuring antigen-specific antibody titers ${ }^{2}$. Large interindividual variation in antibody responses to vaccines administered in early life may limit vaccine effectiveness, leaving some fully vaccinated infants unprotected against serious infectious diseases ${ }^{3}$. Factors that influence vaccine responses include, among others, genetics, sex, perinatal characteristics like gestational age, birth weight, maternal antibodies, and feeding type, but also more general factors like geographical region (reviewed in ${ }^{4}$ ). Recent research has shown that the gut microbiota, i.e. the sum of all microorganisms residing in the human intestinal tract, also plays a role in immune responses to vaccination ${ }^{5-11}$. This offers a potentially modifiable target to improve immunogenicity of childhood vaccines.

The gut microbiome is seeded at birth and rapidly develops over the first months of life under the influence of mode of delivery, breastfeeding, antibiotic administration and nutrition ${ }^{12-15}$. Timely exposure to specific microbes within the critical 'window of opportunity' in early infancy shapes the immune system ${ }^{16-18}$, including the $\mathrm{B}$ cell and immunoglobulin repertoire ${ }^{19,20}$. Microbial imprinting on the immune system may in turn explain part of the variation in vaccine responses. In support of this hypothesis, it has been shown that antibiotic-induced microbial perturbances in an infant mouse model led to impaired antigen-specific immunoglobulin $G(\operatorname{Ig} G)$ responses against five common childhood vaccines ${ }^{21}$. Microbiota perturbance due to antibiotic exposure also resulted in impaired immune responses to seasonal influenza vaccination in healthy adults without preexisting immunity, suggesting that primary responses are more sensitive to microbiota changes than recall responses ${ }^{7}$. In human infants, the composition of the microbial community prevaccination has been correlated with systemic immune responses to oral rotavirus vaccine, oral poliovirus vaccine, Bacillus Calmette-Guérin, hepatitis B, and tetanus vaccines ${ }^{5,6,10,11,22,23}$. However, the temporal relationship between 1. early-life exposures, 2. gut microbiota composition, and 3. subsequent childhood vaccine responses has not yet been studied. 
medRxiv preprint doi: https://doi.org/10.1101/2021.09.01.21262898; this version posted September 3, 2021. The copyright holder for this preprint

(which was not certified by peer review) is the author/funder, who has granted medRxiv a license to display the preprint in perpetuity.

It is made available under a CC-BY 4.0 International license .

Here, we demonstrate in a healthy birth cohort that mode of delivery-associated differences in early-life gut microbial colonization patterns are associated with antigen-specific $\operatorname{IgG}$ responses to pneumococcal and meningococcal conjugate vaccination in saliva. These findings are key for the design of intervention strategies that modulate the gut microbiota to enhance vaccine immunogenicity in infants. 


\section{Methods}

Study design and participants

Fecal samples, saliva and questionnaires were collected from 120 healthy, full-term infants who participated in a prospective birth cohort study, where we previously reported a significant effect of mode of delivery on the gut microbiota in the first months of life ${ }^{24}$. Details on study design were previously published ${ }^{24,25}$. For the current analyses, we expanded our dataset with data and samples up to 18 months from $78(65 \%)$ subjects, who participated in the follow-up study beyond the first year of life. Both parents provided written informed consent. Ethical approval was granted by the Dutch national ethics committee.

Study visits were conducted within 2 hours post-partum, 24-36 hours after birth, at 7 and 14 days and at 1,2, 4, 6, 9, 12 months and, for those who participated in the follow-up study, 18 months of age. Saliva for antibody measurement was collected at the ages of 12 and 18 months. Fecal samples for gut microbiota profiling were collected by the parents prior to each visit, and were directly stored in the home freezer. Details on sample collection are in the appendix. Saliva and feces were transported on dry ice and stored at $-80^{\circ} \mathrm{C}$.

Extensive questionnaires including vaccination dates were collected. Infants were vaccinated according to the Dutch national immunization program (NIP). Ten-valent pneumococcal conjugate vaccine (PCV-10) was administered to infants born before September 2013 (52/120 participants) at the ages of 2, 3, 4, and 11 months, and to infants born from September 2013 (68/120 participants) at the ages of 2, 4, and 11 months due to changes in the NIP. Meningococcus type C (MenC) conjugate vaccination was administered at the age of 14 months.

\section{Laboratory procedures}

Antigen-specific $\operatorname{IgG}$ against the capsular polysaccharides of pneumococcal vaccine serotypes 1, 4, $5,6 \mathrm{~B}, 7 \mathrm{~F}, 9 \mathrm{~V}, 14,18 \mathrm{C}, 19 \mathrm{~F}$, and $23 \mathrm{~F}$ was measured in saliva obtained at 12 months of age, and IgG against MenC polysaccharide in saliva obtained at 18 months of age. Antibodies were quantified using fluorescent bead-based multiplex immunoassays (MIA) as previously described ${ }^{26-}$ 
${ }^{28}$ (appendix). $\mathrm{IgG}$ concentrations below the lower limit of detection, which ranged from $0.08 \mathrm{ng} / \mathrm{ml}$ for pneumococcal serotype 4 to $0.37 \mathrm{ng} / \mathrm{ml}$ for pneumococcal serotype 14 , and was $0.21 \mathrm{ng} / \mathrm{ml}$ for MenC, were set at half the lower limit of detection.

Laboratory processing and microbiota profiling of fecal samples was previously described ${ }^{24}$. In short, bacterial DNA was extracted using a combination of mechanical and chemical lysis methods, and was quantified by quantitative (q)PCR targeting the 16S rRNA gene ${ }^{29}$. The V4 hypervariable region of the 16S rRNA gene was amplified, and amplicon pools were sequenced on the Illumina MiSeq platform (Illumina, San Diego, CA) along with isolation and PCR blanks as negative controls. Sequences were processed in our previously described bioinformatics pipeline ${ }^{30}$. This resulted in an abundance-filtered data set containing only operational taxonomic units (OTU) that represented at least $0.1 \%$ of all reads in at least two samples (623/6690 OTUs). Taxonomic annotations of the 16S rRNA gene sequences were validated using shotgun metagenomics (subset of 20 samples), and species-specific qPCR (all samples) ${ }^{24}$.

\section{$\underline{\text { Statistical analyses }}$}

Data analysis was performed in $\mathrm{R}$ version 4.0.3. All statistical tests were two-tailed, and p-values below 0.050 or Benjamini-Hochberg adjusted p-values below 0.100 was considered statistically significant. All analyses were performed using log-transformed IgG concentrations, and were adjusted for time between vaccination and saliva collection using a second degree polynomial to account for the natural kinetics of the antibody response.

Concordance between IgG concentrations was evaluated using Spearman's rank-order correlations. IgG geometric mean concentrations (GMCs) were compared using ANOVA with post-hoc Tukey tests. Associations between early-life host or microbiota characteristics and IgG concentrations were assessed using multivariable linear models.

Gut microbiota alpha diversity was assessed by the number of observed species, and the Shannon diversity index $\left(\right.$ phylose $\left.^{31}\right)$. Stability of the microbial community composition over time was calculated as the Bray-Curtis (BC) similarity (1-BC dissimilarity) between consecutive samples 
within individuals. Dirichlet multinomial mixture models were used to group infants into community state types (CSTs) based on gut microbiota composition (DirichletMultinomial ${ }^{32}$ ) (appendix). Differences in the gut microbial community composition according to CST were evaluated using permutational analysis of variance (PERMANOVA) $\left(\right.$ vegan $\left.^{33}\right)$. Smoothing-spline analysis of variance (metagenomeSeq-package ${ }^{34,35}$ ) was used to detect differences in individual OTU abundances (present in at least $10 \%$ of samples) over time between infants with responses above and below the median antigen-specific IgG concentration. This method detects differentially abundant OTUs, and identifies the time intervals in which significant differences exist.

$\underline{\text { Role of the funding source }}$

The study funders had no role in study design; data collection, analysis, or interpretation; or writing of the report. The corresponding author had full access to all study data and final responsibility for the decision to submit for publication. 


\section{Results}

We investigated associations between early-life exposures, gut microbiota development in the first year of life, and subsequent antibody responses against pneumococcal and meningococcal conjugate vaccination in 120 healthy, full-term infants. Basic, lifestyle and environmental characteristics were previously published ${ }^{24}$, and are briefly summarized in Table 1. Follow-up of the infants and sample inclusion for IgG measurement are shown in Figure S1. Serotype-specific anti-pneumococcal IgG concentrations in saliva were measured in 101/120 (84.2\%) infants at the age of 12 months (median 28 days [IQR 21-33] after the PCV-10 booster dose). Anti-MenC IgG concentrations in saliva were measured in 66/78 (84.6\%) infants at the age of 18 months (median 116 days [IQR 105-120] after MenC vaccination). Geometric mean concentrations (GMC) of anti-pneumococcal IgG ranged between $7.33 \mathrm{ng} / \mathrm{ml}(95 \%$ CI $5.75-9.33 \mathrm{ng} / \mathrm{ml})$ for serotype $23 \mathrm{~F}$ and $27.30 \mathrm{ng} / \mathrm{ml}(95 \%$ CI $22.14-$ 33.67) for serotype 19F. The anti-MenC IgG GMC was $10.64 \mathrm{ng} / \mathrm{ml}(95 \%$ CI $8.64-13.11 \mathrm{ng} / \mathrm{ml})$ (Figure 1A). $\operatorname{IgG}$ concentrations against the 10 pneumococcal vaccine serotypes strongly correlated with each other (Spearman's rho 0.53-0.84, adjusted $\mathrm{p}<0.001$ for all pairwise correlations), and not with anti-MenC IgG antibodies (Spearman's rho 0.14-0.30, adjusted p>0.469 for all pairwise correlations) (Figure 1B). As serotype-specific anti-pneumococcal IgG concentrations were strongly correlated, we focused our analyses on serotype $6 \mathrm{~B}$, which shows relatively weak antigenic properties, and is commonly found during (severe) pneumococcal disease ${ }^{36}$. Significant findings were validated for the other serotypes.

\section{$\underline{\text { Mode of delivery was associated with vaccine responses }}$}

We first investigated whether early-life host characteristics previously associated with differences in gut microbiome development and/or vaccine immunogenicity, were related to anti-Ps6B and antiMenC IgG responses. Mode of delivery, feeding type, sex, antibiotics use in the first 3 months of life, and pets in the household were related to vaccine responses against one or more serotype, while having older siblings, and daycare attendance were not. These variables were included in multivariable linear models, including an interaction term between mode of delivery and feeding type due to the interdependency of these variables. Natural delivery (in contrast to caesarean (C-)section birth) was 
independently associated with higher anti-Ps6B IgG concentrations $(\beta=0.51$ [95\% CI 0.043-0.97], $\mathrm{p}=0.033$; Figure 2A). However, we also observed a negative interaction between natural delivery and exclusive formula feeding on anti-Ps6B responses $(\beta=-1.32$ [95\% CI $-2.43--0.21], p=0.021$, suggesting that the positive effect of natural birth was diminished by subsequent formula feeding. Similar associations were found for IgG responses to most of the other pneumococcal vaccine serotypes (Table S1). Stratified analyses confirmed that, within the breastfed group, the anti-Ps6B IgG GMC of naturally born infants ( $\mathrm{n}=51)$ was two-fold higher compared to C-section born infants $(\mathrm{n}=33$; adjusted $\mathrm{p}=0.067$ ); similarly, within the naturally born group, the anti-Ps6B IgG GMC of breastfed infants $(n=51)$ was 3.5 -fold higher compared to formula fed infants $(n=7$; adjusted $p=0.083$ ). AntiPs6B IgG concentrations did not differ between feeding types within the C-section born group (Figure 2B).

Likewise, for MenC, natural delivery was also associated with higher IgG concentrations compared to C-section delivery ( $\beta=0.42$ [95\% CI 0.016-0.83], $\mathrm{p}=0.042$ ), which was independent of feeding type (Figure 2A). In a stratified analysis of breastfed infants, naturally born infants $(n=38)$ showed a 1.5fold higher anti-MenC IgG GMC compared to C-section born infants ( $=18$; adjusted $\mathrm{p}=0.088$; Figure 2B). Sex, antibiotic use, and having pets were not significantly associated with IgG responses against Ps6B or MenC.

Gut microbial community composition at 1 week of age was associated with vaccine responses

We then studied whether gut microbiota development in the first year of life was associated with antiPs6B and anti-MenC IgG responses. Overall, 1052 out of 1177 fecal samples (89.4\%) passed quality control for 16S rRNA gene-based sequencing, and were included in further analyses as previously described $^{24}$.

No association was found between alpha diversity and anti-Ps6B or anti-MenC IgG concentrations at any time point, with the exception of an inverse correlation between the observed number of species at the age of two months and anti-Ps6B IgG concentrations ( $\beta=-0.029$ [95\% CI -0.049- -0.0087], adjusted 
$\mathrm{p}=0.082$ ). This association was not observed for the other pneumococcal vaccine serotypes (data not shown).

Gut microbiota stability between day one and week one, and between week one and week two correlated with higher anti-Ps6B IgG concentrations (day one-week one: $\beta=1.66$ [95\% CI 0.44-2.88], adjusted $\mathrm{p}=0.074$; week one-week two: $\beta=1.22$ [95\% CI 0.22-2.22], adjusted $\mathrm{p}=0.077$ ), which was not observed for any other time interval. Microbiota stability in the first two weeks of life was also significantly positively associated with $\operatorname{IgG}$ concentrations against all other pneumococcal vaccine serotypes (adjusted $\mathrm{p} \leq 0.083$, Table S2). In contrast, no significant associations were found between microbiota stability and anti-MenC IgG concentrations.

The first two weeks of life, where gut microbiota stability was associated with anti-pneumococcal IgG concentrations, is compatible with the time frame when we previously found the largest difference in gut microbial composition between naturally born and C-section born infants in this cohort (at the age of one week $)^{24}$. Therefore, we decided to focus on the microbial community composition in 'week one' samples, where we identified three distinct community state types (CSTs) (Figure S2). These CSTs differed considerably in community composition (PERMANOVA: $\mathrm{R}^{2}=34.8 \%, \mathrm{p}<0.001$ ). Infants with CST1 (n=55) had a microbial community with low abundances of both Bifidobacterium and Escherichia coli, while infants with CST2 $(\mathrm{n}=48)$ had profiles with high Bifidobacterium abundances, and infants with CST3 (n=16) had high E. coli abundances (Figure 3A). Species-level microbial composition of the CSTs was largely confirmed by shotgun sequencing of 20 week one samples (Figure S3).

We then studied whether these CSTs were associated with anti-Ps6B and anti-MenC IgG concentrations following vaccination. Infants with CST1 had the lowest IgG concentrations against both Ps6B and MenC (anti-Ps6B IgG: GMC 7.84 ng/ml [95\% CI 4.88-12.60]; anti-MenC IgG: GMC $8.28 \mathrm{ng} / \mathrm{ml}$ [95\% CI 5.93-11.56]) (Figure 3B). Compared to infants with CST1, anti-Ps6B IgG concentrations were approximately two-fold higher in infants with CST2 (GMC $17.05 \mathrm{ng} / \mathrm{ml}$ [95\% CI 12.64-23.00], adjusted $\mathrm{p}=0.030$ ) as well as in infants with CST3 (GMC $14.85 \mathrm{ng} / \mathrm{ml}$ [95\% CI 7.3629.97], adjusted $\mathrm{p}=0.273$ ), though only the comparison of anti-6B responses between CST1 and CST2 
infants was significant. We observed similar associations between week one CSTs and IgG responses against most other pneumococcal vaccine serotypes (Table S3). By contrast, anti-MenC IgG concentrations were not significantly different between CST groups, although infants with CST3 showed a nearly two-fold higher anti-MenC GMC (15.76 ng/ml [95\% CI 7.25-34.26], adjusted $\mathrm{p}=0.148)$ than infants with CST1.

Mode of delivery was a strong driver of week one CSTs. All infants with CST2 were naturally born, which was significantly more than infants with CST1 (29.1\%; Fisher's exact test, adjusted p<0.001), or CST3 $(62.5 \%$, adjusted $\mathrm{p}<0.001)$. Natural birth was also overrepresented in infants with CST3 compared to CST1 (adjusted $\mathrm{p}=0.020$ ). In contrast, feeding type (breastfeeding vs. exclusive formula feeding) was not significantly different between these CSTs. Interestingly, a post-hoc analysis revealed that the association between mode of delivery and anti-Ps6B IgG responses disappeared with the addition of week one CST as an independent variable, indicating that the positive effect of natural delivery on anti-Ps6B IgG depended fully on the CST. In contrast, natural delivery remained significantly associated with anti-MenC IgG responses, regardless of week one CST, suggesting an independent effect (Table S4).

To evaluate whether observed differences in early-life microbial community composition were sustained for a prolonged time, including time points closer to vaccination, temporal development of the gut microbiota according to week one CST was assessed using PERMANOVA. The microbial community composition of children according to their CST defined at week one converged over time, resulting in no differences between samples belonging to the CST groups from month six onward (Figure 4A). In pairwise comparisons, the observed differences in microbial community composition disappeared between infants with CST1 and CST3 by month one, between infants with CST2 and CST3 by month four, and between infants with CST1 and CST2 by month six. Similarly, relative abundances of Bifidobacterium and E. coli converged over time between CST groups (Figure 4B). At the age of 12 months, we identified two distinct CSTs, which were not significantly associated with anti-Ps6B or anti-MenC IgG responses, confirming that early-life microbiota were more strongly related to vaccine responses than the microbiota close to time of vaccination (Figure S4). 
Early-life dynamics of individual OTUs are related to vaccine responses

Finally, we investigated differences in individual OTU succession patterns within the first two months between high and low vaccine responders (stratified along the median antigen-specific IgG response). Higher abundances of E. coli (days 0-41, adjusted p=0.013) and Bifidobacterium (days 0-5, adjusted $\mathrm{p}=0.027$ ) were associated with high anti-Ps6B responses (confirmed for $7 / 9$ other pneumococcal vaccine serotypes, Table S5). This was also observed for several Bacteroides OTUs, whereas Clostridium, Prevotella and Streptococcus pyogenes were associated with low responses (adjusted $\mathrm{p}<0.050)$.

Higher E. coli abundance (days $0-13$, adjusted p=0.072) was also associated with high anti-MenC responses (Table S6). Because the MenC vaccination is administered at the age of 14 months, which is much later in life than the pneumococcal vaccinations, we extended the analysis to 12 months to allow for identification of associations with OTUs that colonize later in life. In high anti-MenC responders, we observed significantly higher abundances of multiple low abundant OTUs belonging to the Lachnospiraceae family, including Fusicatenibacter saccharivorans (days 101-381, adjusted $\mathrm{p}=0.080$ ), Pseudobutyrivibrio (days 125-381, adjusted $\mathrm{p}=0.036$ ) and several Blautia and Roseburia OTUs (Table S7). 


\section{Discussion}

We studied interactions between early-life exposures, gut microbial community development in the first year of life, and subsequent antibody responses in saliva against pneumococcal and meningococcal conjugate vaccination in a healthy birth cohort. A stable gut microbial community with high abundances of potentially beneficial bacteria in the first weeks of life, including Bifidobacterium and E. coli, was associated with high antibody responses to pneumococcal vaccination at 12 months of life. Furthermore, high E. coli abundance in early life was associated with high antibody responses to meningococcal vaccination at 18 months of life. Natural delivery was associated with high antibody responses to both vaccines, and, as we previously showed in this cohort $^{24}$, with the early-life gut microbiota colonization patterns that we now associated with high antibody responses. Previous studies on associations between gut microbiota composition and serum antibody responses have focused on the microbiota near the time of vaccination ${ }^{5,6,10,11,22}$. However, our findings suggest that especially early-life gut microbiota development may set the stage for immune responses to childhood vaccinations.

Antibody responses to vaccination are elicited through activation of vaccine-specific B cells which will differentiate into immunoglobulin-secreting plasma cells and memory B cells. The gut microbiota have been implicated in the shaping of the systemic B cell and immunoglobulin repertoire in early life $\mathrm{e}^{19,20,37}$. For instance, a deficient production of $\operatorname{IgA}$ and IgG1 in germ free mice can be restored by microbial exposure ${ }^{38}$. In infants, a culture-based study showed that the presence of E. coli and bifidobacteria in the gut in the first weeks of life was related to higher numbers of circulating CD27 $7^{+}$memory B cells at four and 18 months of life ${ }^{39}$. This suggests that bacterial colonization patterns in early infancy drive B cell maturation, and have a lasting effect on immunity $^{39}$. In line with this observation, we found associations between gut microbiota community state types (CSTs) characterized by high abundances of E. coli and/or Bifidobacterium in 1-week-old infants and higher antibody responses to vaccination months later in childhood.

Previous studies have provided evidence for a positive effect of E. coli and Bifidobacterium on the immune response to vaccination. For instance, higher abundances of Gram-negatives including $E$. 
coli were associated with an adequate immune response against oral rotavirus vaccines ${ }^{5}$. Another study showed that treatment with the probiotic E. coli Nissle in a pig model enhanced the immune response to human rotavirus infection ${ }^{40}$, providing a causal link. A potential mechanism whereby E. coli may influence vaccine responses was pinpointed by a study demonstrating that impaired antibody responses to seasonal influenza vaccination in germ-free or antibiotic-treated mice were restored through TLR5-signaling by flagellated, but not unflagellated, E. coli ${ }^{8}$, suggesting strainand antigen-specific immune enhancement. Furthermore, early-life absence of Bifidobacterium has been associated with reduced systemic immune responses to Bacillus Calmette-Guérin, polio virus, tetanus and hepatitis $\mathrm{B}$ vaccination ${ }^{11,22}$, which we also found for pneumococcal conjugate vaccination. Lack of early bifidobacterial colonization has been linked to immune dysregulation at the age of three months in a previous study, showing reduced levels of circulating plasmablasts, and naïve and transitional $\mathrm{B}$ cells ${ }^{17}$. Although the exact mechanisms remain to be unraveled, very early-life microbiota-host crosstalk at the intestinal mucosa appears to imprint on systemic immunity, including vaccine responses.

Mode of delivery and breastfeeding are important drivers of early-life Bifidobacterium and E. coli abundance ${ }^{13,24,41}$, whereas antibiotic treatment in the neonatal period has shown to dramatically reduce these bacteria ${ }^{42}$. Our results suggest that early-life microbiota mediate the relationship between mode of delivery and anti-pneumococcal vaccine responses, further emphasizing the importance of discouraging the increasing application of C-section in the absence of medical urgency to preserve the microbiota-immune axis in infants. Similarly, antibiotic-induced microbiota disruption may lead to reduced vaccine responses ${ }^{7,21}$. Preterm infants have also been shown to generate lower antibody levels following vaccination compared to term-born controls ${ }^{43}$. In our healthy, term-born cohort, very few infants required antibiotic treatment in the first weeks of life, and further studies are required to compare our findings to (preterm) infants who received antibiotics as neonates.

We observed stronger associations of specific gut colonization patterns in early life with antibody responses to pneumococcal vaccination than with antibody responses to meningococcal 
vaccination. Furthermore, antibody responses against pneumococcal serotypes were not correlated to those against MenC, suggesting that early-life microbe-mediated immune modulation might be antigen-specific. A more likely explanation is that pneumococcal and meningococcal vaccinations are administered at different ages. When meningococcal vaccination is administered at 14 months of age, the immune system has been exposed to other factors, and is already more mature and possibly more resilient to microbiota-related cues than when the first pneumococcal vaccination is administered at two months of age ${ }^{16}$. Notably, we associated higher abundances of members of the Lachnospiraceae family, including butyrate-producing taxa, with higher anti-meningococcal antibody responses. The abundance of these bacteria in the gut typically increases following the cessation of breastfeeding ${ }^{41,44}$, and are generally found to be beneficial for the developing immune system $^{45}$.

Our findings lay a foundation for studies investigating interventions that modulate the infant gut microbiota to improve vaccine immunogenicity. Perturbed gut microbial colonization patterns likely contribute to reduced vaccine effectiveness across certain populations and settings ${ }^{9}$. Methods to modulate the gut microbiota following perturbations such as C-section birth are being investigated, and range from probiotic administration ${ }^{46}$ to maternal fecal microbiota transplants ${ }^{47}$, but it remains unknown if such interventions confer any long-term health benefits including enhanced vaccine immunogenicity. Our findings also suggest that different interventions may be indicated for vaccinations given earlier in life compared to later in life, and this should be considered in future studies.

Strengths of our work include the dense sampling at different timepoints, especially in the beginning of life. The extensively documented epidemiological data and microbiota composition of our cohort allowed us to establish associations between gut microbiota and vaccine responses in healthy infants. Furthermore, with the sensitive MIA technology, we could accurately measure antigen-specific antibody concentrations, even in very low volumes of saliva. Limitations of our work include using saliva for antibody measurements rather than serum. However, IgG concentrations in saliva were shown to correlate with serum concentrations ${ }^{48}$, and are, therefore, a 
valid proxy for systemic IgG. Furthermore, while pneumococcal and meningococcal vaccination protect from infection primarily through neutralizing $\mathrm{IgG}$, we did not assess other parameters of immunity such as IgA, antibody affinity, and T cell responses. Future studies could employ a systems biology approach to obtain a complete overview of the mechanisms that underlie interindividual variation in vaccine responses ${ }^{2,49}$. Our observational study was also not primarily designed to study relationships between drivers, microbes and health outcomes such as antibody responses to vaccination, which limited our power to detect significant associations. Finally, the time between vaccination of the infants and antibody measurement was variable, which we corrected for in our analyses, but may still have affected our results.

In conclusion, we demonstrate that mode of delivery-associated differences in the gut microbiota in the first weeks of life, including differences in E. coli and Bifidobacterium abundances, are associated with anti-pneumococcal and anti-MenC $\operatorname{IgG}$ responses to vaccination. Incorporating antibody responses to vaccination as a parameter in future trials of early-life microbiota modulation could offer opportunities to assess beneficial outcomes on the microbe-mediated training of the immune system. Improved understanding of the microbial factors driving immune maturation and vaccine immunogenicity is key to improve vaccine performance and combat infectious diseases in children. 


\section{References}

1. World Health Organization. State of the world's vaccines and immunization. 2009. https://apps. who.int/iris/bitstream/handle/10665/44169/9789241563864_eng.pdf;jsessionid=82 C5A470414517FAF8840256D1A47D99?sequence=1 (date accessed May 1, 2021).

2. Pulendran, B. \& Ahmed, R. Immunological mechanisms of vaccination. Nature Immunology 12, 509-517 (2011).

3. Grassly, N. C., Kang, G. \& Kampmann, B. Biological challenges to effective vaccines in the developing world. Philosophical Transactions of the Royal Society B: Biological Sciences 370, (2015).

4. Zimmermann, P. \& Curtis, N. Factors That Influence the Immune Response to Vaccination. Clinical Microbiology Reviews 32, e00084-18 (2019).

5. Harris, V. et al. Rotavirus vaccine response correlates with the infant gut microbiota composition in Pakistan. Gut Microbes 9, 93-101 (2017).

6. Harris, V. C. et al. Significant Correlation Between the Infant Gut Microbiome and Rotavirus Vaccine Response in Rural Ghana. The Journal of Infectious Diseases 215, 34-41 (2017).

7. Hagan, T. et al. Antibiotics-Driven Gut Microbiome Perturbation Alters Immunity to Vaccines in Humans. Cell 178, 1313-1328.e13 (2019).

8. Oh, J. Z. et al. TLR5-mediated sensing of gut microbiota is necessary for antibody responses to seasonal influenza vaccination. Immunity 41, 478-492 (2014).

9. de Jong, S. E., Olin, A. \& Pulendran, B. The Impact of the Microbiome on Immunity to Vaccination in Humans. Cell Host and Microbe 28, 169-179 (2020).

10. Nazmul Huda, M. et al. Stool Microbiota and Vaccine Responses of Infants. Pediatrics 134, e362-e372 (2014).

11. Nazmul Huda, M. et al. Bifidobacterium Abundance in Early Infancy and Vaccine Response at 2 Years of Age. Pediatrics 143, e20181489 (2019).

12. Subramanian, S. et al. Persistent gut microbiota immaturity in malnourished Bangladeshi children. Nature 510, 417-421 (2014).

13. Bokulich, N. A. et al. Antibiotics, birth mode, and diet shape microbiome maturation during early life. Science Translational Medicine 8, 343ra82 (2016).

14. Jakobsson, H. E. et al. Decreased gut microbiota diversity, delayed Bacteroidetes colonisation and reduced Th1 responses in infants delivered by Caesarean section. Gut 63, 559-566 (2014).

15. Mitchell, C. et al. Delivery Mode Affects Stability of Early Infant Gut Microbiota. Cell Reports Medicine 1, 100156 (2021).

16. Olin, A. et al. Stereotypic Immune System Development in Newborn Children. Cell 174, 12771292.e14 (2018).

17. Henrick, B. M. et al. Bifidobacteria-mediated immune system imprinting early in life. Cell 184, $1-15(2021)$.

18. Fujimura, K. E. et al. Neonatal gut microbiota associates with childhood multi- sensitized atopy and T-cell differentiation. Nat Med 22, 1187-1191 (2016). 
19. Li, H. et al. Mucosal or systemic microbiota exposures shape the B cell repertoire. Nature 584, (2020).

20. New, J. S. et al. Neonatal Exposure to Commensal-Bacteria-Derived Antigens Directs Polysaccharide-Specific B-1 B Cell Repertoire Development. Immunity 53, 1-15 (2020).

21. Lynn, M. A. et al. Early-Life Antibiotic-Driven Dysbiosis Leads to Dysregulated Vaccine Immune Responses in Mice. Cell Host and Microbe 23, 653-660.e5 (2018).

22. Mullié, C. et al. Increased Poliovirus-Specific Intestinal Antibody Response Coincides with Promotion of Bifidobacterium longum-infantis and Bifidobacterium breve in Infants: A Randomized, Double-Blind, Placebo-Controlled Trial. Pediatric Research 56, 791-795 (2004).

23. Fix, J. et al. Association between gut microbiome composition and rotavirus vaccine response among Nicaraguan infants. American Journal of Tropical Medicine and Hygiene 102, 213-219 (2020).

24. Reyman, M. et al. Impact of delivery mode-associated gut microbiota dynamics on health in the first year of life. Nature Communications 10, 4997 (2019).

25. Bosch, A. A. T. M. et al. Development of Upper Respiratory Tract Microbiota in Infancy is Affected by Mode of Delivery. EBioMedicine 9, 336-345 (2016).

26. Elberse, K. E. M., Tcherniaeva, I., Berbers, G. A. M. \& Schouls, L. M. Optimization and application of a multiplex bead-based assay to quantify serotype-specific IgG against streptococcus pneumoniae polysaccharides: Response to the booster vaccine after immunization with the pneumococcal 7-valent conjugate vaccine. Clinical and Vaccine Immunology 17, 674-682 (2010).

27. de Voer, R. M. et al. Development of a fluorescent-bead-based multiplex immunoassay to determine immunoglobulin $\mathrm{G}$ subclass responses to Neisseria meningitidis serogroup A and C polysaccharides. Clinical and Vaccine Immunology 15, 1188-1193 (2008).

28. Pickering, J. W. et al. A Multiplexed Fluorescent Microsphere Immunoassay for Antibodies to Pneumococcal Capsular Polysaccharides. American Journal of Clinical Pathology 117, 589596 (2002).

29. Biesbroek, G. et al. Deep Sequencing Analyses of Low Density Microbial Communities: Working at the Boundary of Accurate Microbiota Detection. PLOS ONE 7, e32942 (2012).

30. Bosch, A. A. T. M. et al. Maturation of the Infant Respiratory Microbiota, Environmental Drivers, and Health Consequences. A Prospective Cohort Study. American Journal of Respiratory and Critical Care Medicine 196, 1582-1590 (2017).

31. McMurdie, P. J. \& Holmes, S. Phyloseq: An R Package for Reproducible Interactive Analysis and Graphics of Microbiome Census Data. PLOS ONE 8, e61217 (2013).

32. Holmes, I., Harris, K. \& Quince, C. Dirichlet Multinomial Mixtures: Generative Models for Microbial Metagenomics. PLoS ONE 7, 30126 (2012).

33. Oksanen, J. et al. vegan: Community Ecology Package. (2017). https://CRAN.Rproject.org/package $=$ vegan

34. Paulson, J. N., Talukder, H. \& Bravo, H. C. Longitudinal differential abundance analysis of microbial marker-gene surveys using smoothing splines. bioRxiv (2017) doi:10.1101/099457. 
35. Paulson, J. N., Stine, O. C., Bravo, H. C. \& Pop, M. Differential abundance analysis for microbial marker-gene surveys. Nature Methods 10, 1200-1202 (2013).

36. Kalin, M. Pneumococcal serotypes and their clinical relevance. Thorax 53, 159-162 (1998).

37. Zeng, M. Y. et al. Gut Microbiota-Induced Immunoglobulin G Controls Systemic Infection by Symbiotic Bacteria and Pathogens. Immunity 44, 647-658 (2016).

38. Gensollen, T., Iyer, S. S., Kasper, D. L. \& Blumberg, R. S. How colonization by microbiota in early life shapes the immune system. Science vol. 352 539-544 (2016).

39. Lundell, A.-C. et al. Infant B Cell Memory Differentiation and Early Gut Bacterial Colonization. The Journal of Immunology 188, 4315-4322 (2012).

40. Michael, H. et al. Escherichia coli Nissle 1917 administered as a dextranomar microsphere biofilm enhances immune responses against human rotavirus in a neonatal malnourished pig model colonized with human infant fecal microbiota. PLOS ONE 16, e0246193 (2021).

41. Stewart, C. J. et al. Temporal development of the gut microbiome in early childhood from the TEDDY study. Nature 562, 583-588 (2018).

42. Gasparrini, A. J. et al. Persistent metagenomic signatures of early-life hospitalization and antibiotic treatment in the infant gut microbiota and resistome. Nature Microbiology 4, 22852297 (2019).

43. Rouers, E. D. M. et al. Association of Routine Infant Vaccinations with Antibody Levels among Preterm Infants. JAMA - Journal of the American Medical Association 324, 1068-1077 (2020).

44. Tsukuda, N. et al. Key bacterial taxa and metabolic pathways affecting gut short-chain fatty acid profiles in early life. The ISME Journal in press, (2021).

45. Depner, M. et al. Maturation of the gut microbiome during the first year of life contributes to the protective farm effect on childhood asthma. Nature Medicine (2020) doi:10.1038/s41591020-1095-x.

46. Alcon-Giner, C. et al. Microbiota Supplementation with Bifidobacterium and Lactobacillus Modifies the Preterm Infant Gut Microbiota and Metabolome: An Observational Study. Cell Reports Medicine 1, 100077 (2020).

47. Korpela, K. et al. Maternal Fecal Microbiota Transplantation in Cesarean-Born Infants Rapidly Restores Normal Gut Microbial Development: A Proof-of-Concept Study. Cell 1-11 (2020).

48. Rodenburg, G. D. et al. Salivary Immune Responses to the 7-Valent Pneumococcal Conjugate Vaccine in the First 2 Years of Life. PLoS ONE 7, 1-8 (2012).

49. Li, S. et al. Molecular signatures of antibody responses derived from a systems biology study of five human vaccines. Nature Immunology 15, 195-204 (2014). 


\section{Acknowledgements}

The authors are indebted to all the participating children and their families. We thank all the members of the research team of the Spaarne Gasthuis Academy, the laboratory staff, and the Streeklaboratorium Haarlem. We are grateful to Belinda van 't Land from Nutricia for providing some of the reagents.

\section{Declaration of interests}

None of the authors have a conflict of interest to disclose.

\section{Author contributions}

D.B., M.A. van H., and E.A.M.S conceived and designed the study. M.A. van H. was involved in enrolling the participants. M.L.J.N.C., F. de H., and G.A.M.B. were responsible for the execution and quality control of the laboratory work. E.M. de K., M.R., D.B., and S.F. analyzed the data. E.M. de K.,

D. van B., M.A. van H., E.A.M.S., D.B., and S.F. wrote the paper. All authors significantly contributed to interpreting the results, critically revised the manuscript for important intellectual content, and approved the final manuscript. E.M. de K., M.R., and M.L.J.N.C. have verified the microbiome and participant data. E.M. de K., and F. de H. have verified the antibody data.

\section{Data availability}

Sequence data that support the findings of this study have been deposited in the NCBI Sequence Read Archive (SRA) database with BioProject ID PRJNA481243, and PRJNA555020. Deidentified participant data including antibody measurements, and data dictionaries can be made available after approval of a proposal. 
medRxiv preprint doi: https://doi.org/10.1101/2021.09.01.21262898; this version posted September 3, 2021. The copyright holder for this preprint (which was not certified by peer review) is the author/funder, who has granted medRxiv a license to display the preprint in perpetuity.

\section{It is made available under a CC-BY 4.0 International license.}

\section{Figures}

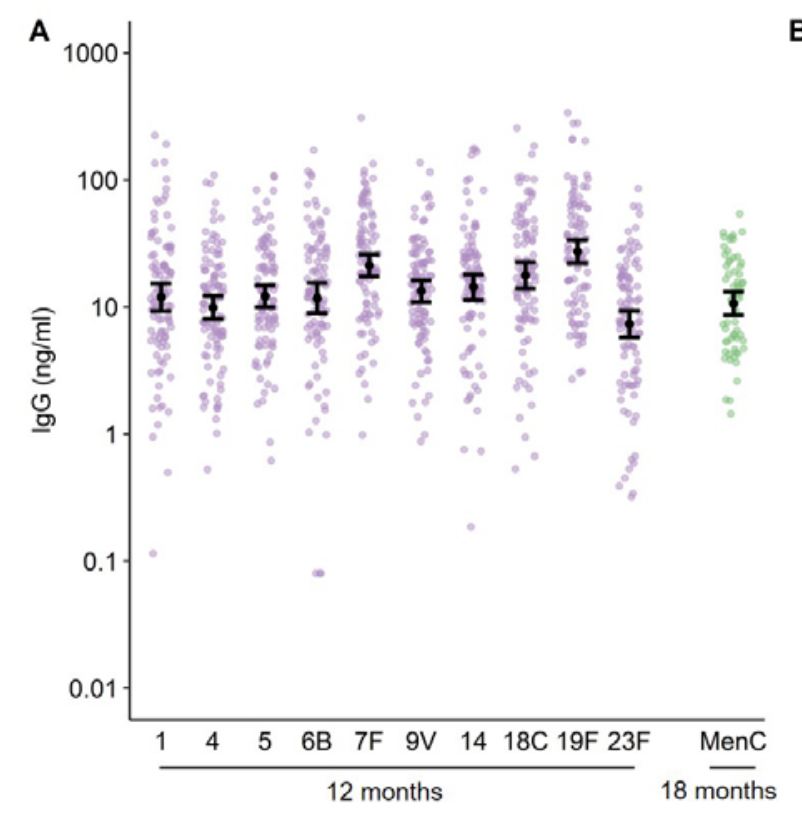

B

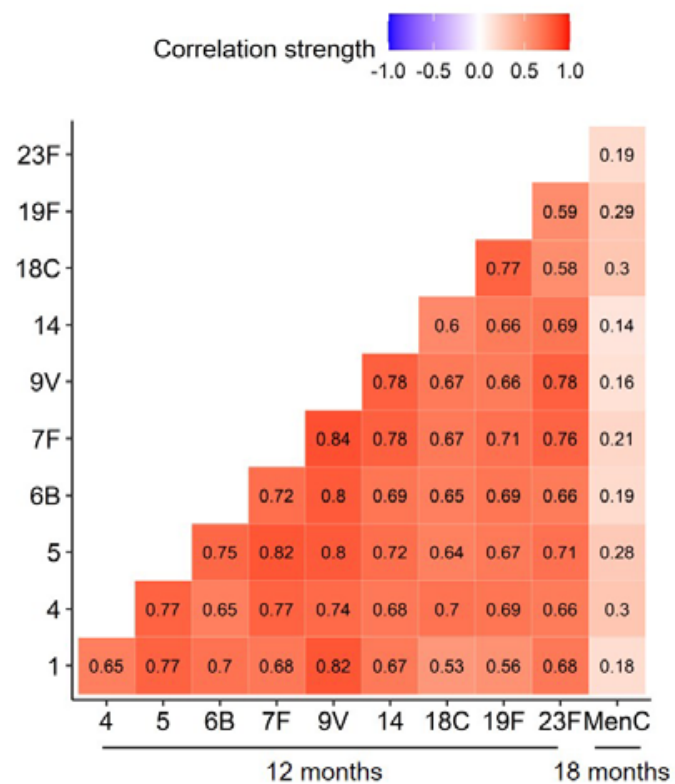

Figure 1. Anti-pneumococcal and anti-MenC IgG concentrations following vaccination.

A) IgG concentrations against 10 pneumococcal vaccine serotypes $(1,4,5,6 \mathrm{~B}, 7 \mathrm{~F}, 9 \mathrm{~V}, 14,18 \mathrm{C}, 19 \mathrm{~F}$ and $23 \mathrm{~F})$ and meningococcus type $\mathrm{C}(\mathrm{MenC})$ following vaccination. Black dots and error bars represent geometric mean concentrations with $95 \%$ confidence intervals. B) Correlation plot of IgG concentrations against the 10 pneumococcal vaccine serotypes and against MenC following vaccination. Numbers indicate the correlation strength, which was evaluated using Spearman's correlation coefficients. 

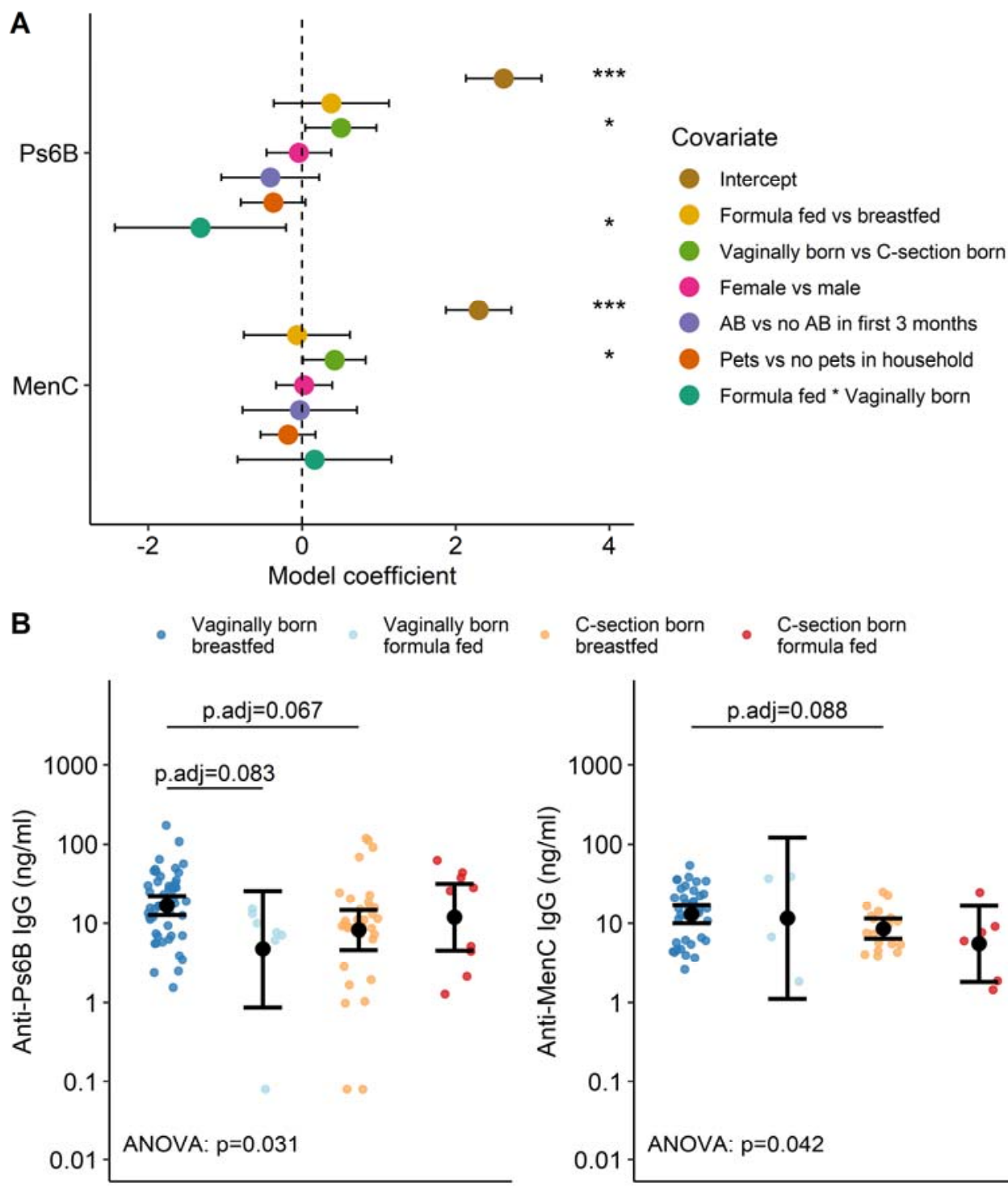

Figure 2. Associations between early-life exposures and anti-pneumococcal and anti-MenC IgG concentrations following vaccination.

A) Colored dots and error bars represent coefficients with $95 \%$ CI of a multivariable linear model with logtransformed $\mathrm{IgG}$ concentrations as dependent variable. Significance is indicated by *: $\mathrm{p}<0.05$; **: $\mathrm{p}<0.005$; or ***: $\mathrm{p}<0.001$. Abbreviations: $\mathrm{C}$-section=caesarean section; $\mathrm{AB}=$ antibiotics. $\mathrm{B})$ post-vaccination antipneumococcal serotype 6B (anti-Ps6B) IgG concentrations (left) and post-vaccination anti-meningococcus type $\mathrm{C}$ (anti-MenC) IgG concentrations (right) in infants stratified according to mode of delivery (vaginal birth vs. Csection) and feeding type (breastfeeding vs. exclusive formula feeding from birth). Black dots and error bars represent geometric mean concentrations with $95 \%$ CI. Significance was assessed using analysis of variance (ANOVA) on log-transformed IgG concentrations followed by a post-hoc Tukey test adjusting for multiple comparisons using the Benjamini-Hochberg procedure, also correcting for time between vaccination and IgG measurements. 
medRxiv preprint doi: https://doi.org/10.1101/2021.09.01.21262898; this version posted September 3, 2021. The copyright holder for this preprint (which was not certified by peer review) is the author/funder, who has granted medRxiv a license to display the preprint in perpetuity.

\section{It is made available under a CC-BY 4.0 International license.}

A OTU

Binidobacterium (1) Escherichia cali (2) Staphyiococcus (3) Klebsiella (4)

Enteracoccus faecium (s)

Streptocaccus salivarius (6)

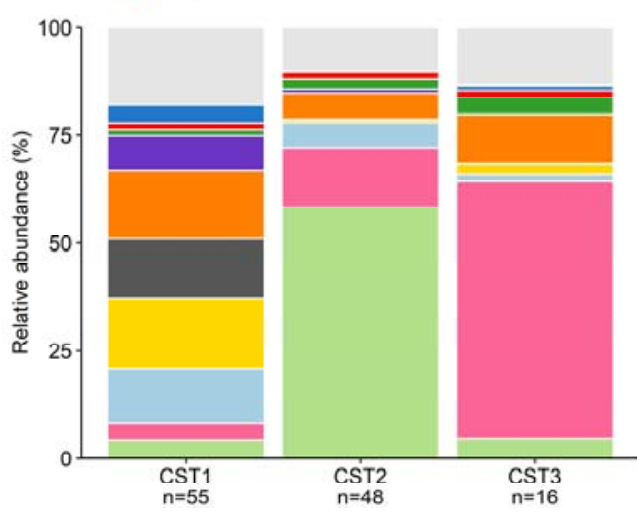

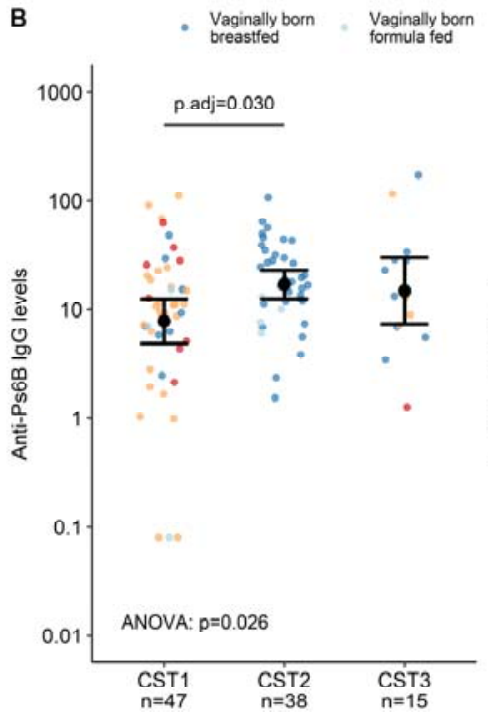

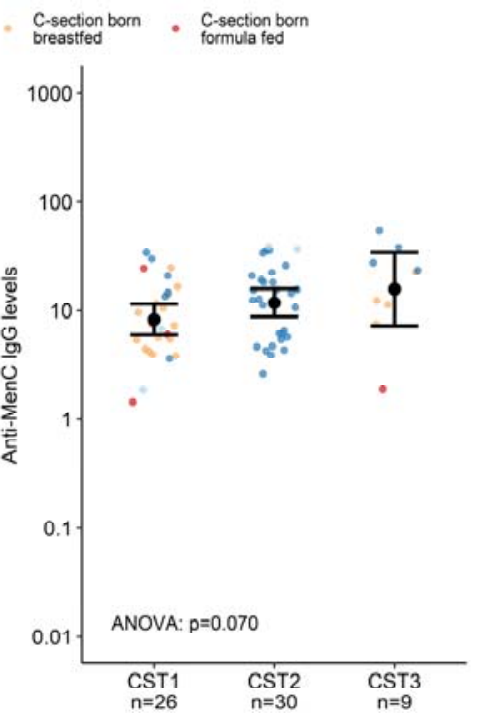

Figure 3. Gut microbial community state types at week 1 and anti-Ps6B and anti-MenC IgG concentrations.

(A) Relative abundances of the top 10 OTUs per community state type (CST) defined at 1 week of age. (B) CSTs are plotted against anti-Ps6B IgG concentrations as representative pneumococcal serotype (left) and antiMenC IgG concentrations (right). Dots are colored according to mode of delivery and feeding type from birth. Black dots and error bars represent geometric mean concentrations with $95 \%$ confidence intervals. Significance was assessed using ANOVA on log-transformed IgG concentrations followed by post-hoc Tukey tests adjusting for multiple comparisons using the Benjamini-Hochberg procedure, also correcting for time between vaccination and IgG measurements. Abbreviations: OTU = operational taxonomic unit; $\mathrm{p}$.adj = adjusted p-value. 
A

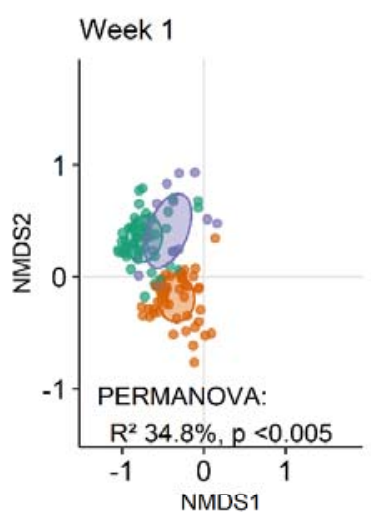

Month 4

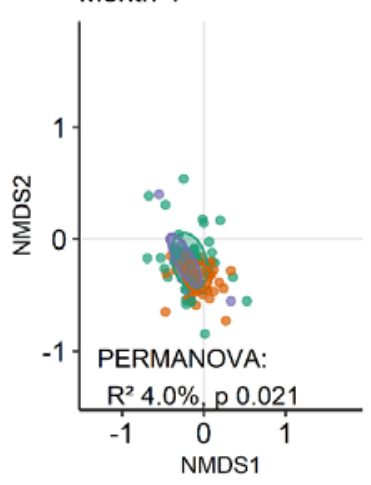

B

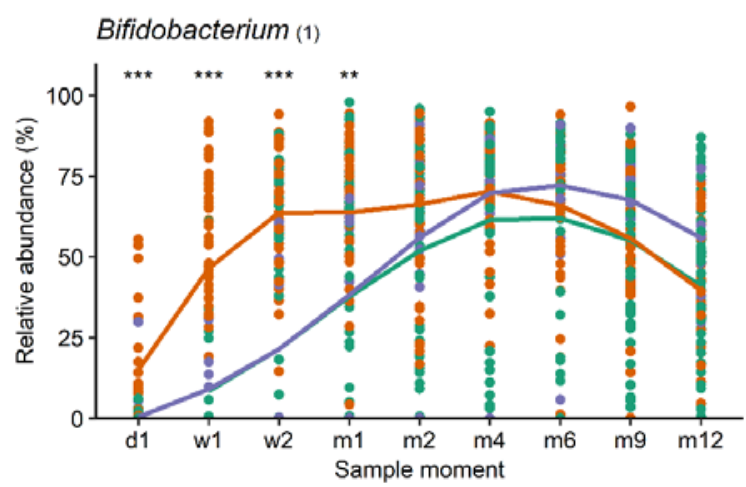

CST $\bullet 1 \div 2 \square 3$
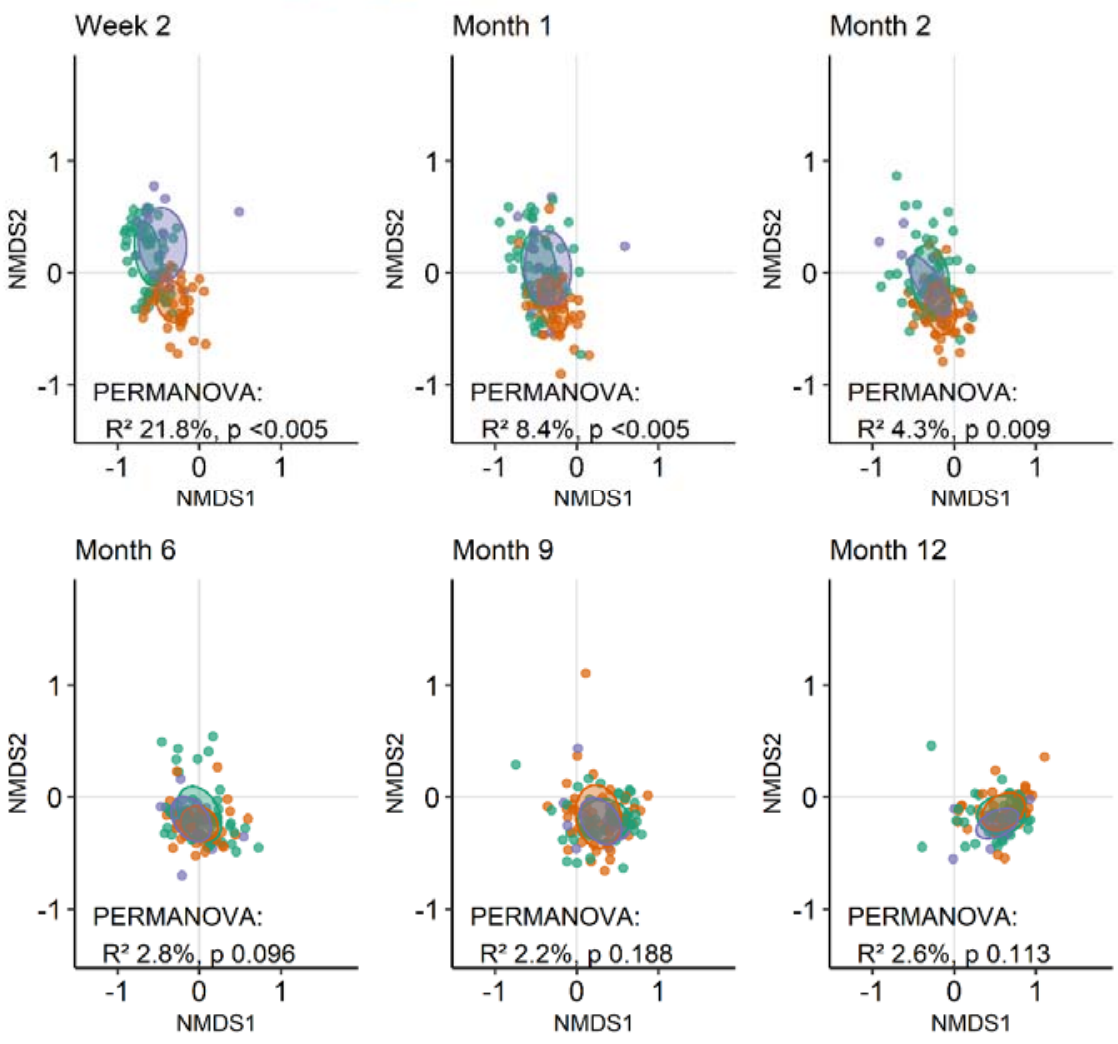

Month 12

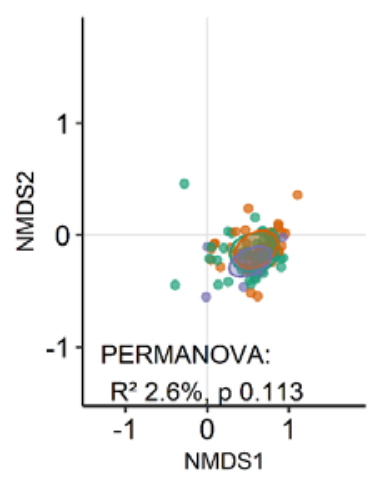

Figure 4. Temporal gut microbial composition development according to week 1 CST.

(A) Non-metric multidimensional scaling plots based on Bray-Curtis dissimilarity, depicting the gut microbial composition per timepoint. Each dot represents the microbiota composition in a single participant's sample. Infants are stratified according to week 1 community state type (CST). Ellipses represent the standard deviation of data points for each CST. Significance of differences according to week 1 CST was assessed using permutational analysis of variance (PERMANOVA). (B) Relative abundances of Bifidobacterium (1) (left) and Escherichia coli (2) (right) over time according to week 1 CST. Significance of differences according to week 1 CST was assessed using Kruskal Wallis tests and indicated by: *** for $\mathrm{p}<0.001$, ** for $\mathrm{p}<0.005$, and $*$ for $\mathrm{p}<0.05$. 
medRxiv preprint doi: https://doi.org/10.1101/2021.09.01.21262898; this version posted September 3, 2021. The copyright holder for this preprint (which was not certified by peer review) is the author/funder, who has granted medRxiv a license to display the preprint in perpetuity.

It is made available under a CC-BY 4.0 International license.

\section{Tables}

Table 1. Cohort description.

\begin{tabular}{r|c}
$\mathrm{n}$ & 120 \\
\hline Sex, female (\%) & $63(52.5)$ \\
Mode of delivery, vaginal (\%) & $74(61.7)$ \\
Exclusive formula feeding from birth (\%) & $22(18.3)$ \\
Breastfeeding, number of days (median [IQR]) & $72.50[3.00,236.75]$ \\
Presence of siblings (\%) & $83(69.2)$ \\
Number of siblings (median [IQR]) & $1.00[0.00,1.00]$ \\
Pets in the household $(\%)$ & $54(45.0)$ \\
Antibiotics in first 3 months of life $(\%)$ & $14(11.7)$
\end{tabular}

